\title{
Error analysis of air traffic control command decision based on rough complex network
}

\author{
Wang Xinyu ${ }^{1,2}$, Cui Haolin², Yang Jie ${ }^{2}$ \\ 1.School of People's Liberation Army Air Force Harbin Flight Academy, Harbin Heilongjiang, China \\ 150000 \\ 2.College of Air Force Engineering University Air Traffic Control and Navigation College, Xi'an, \\ Shaanxi, China 710051
}

Keywords: air traffic control; multiple constraints and decision-making method; complex network; rough set

\begin{abstract}
Air traffic control is also named flight control and it is the general term of supervision control and mandatory management implemented by relevant departments according to flight rules issued by the country for aircrafts flying in the sky. The main purpose is to maintain flight order and prevent collision of aircrafts and collision between aircrafts with barriers on the ground. Attribute association degree is combined with cost-sensitive ideology in the Thesis and one method to generate air traffic control tree based on complex network with compound rough set integration. In selecting splitting attributes, the attribute association degree is not considered in the method and the cost-sensitive ideology is also introduced. The experiment result shows that the decision-making tree constructed with the method in the Thesis has higher classification accuracy and fewer quantity of nodes.
\end{abstract}

\section{Introduction}

Air traffic control is also named flight control and it is the general term of supervision control and mandatory management implemented by relevant departments according to flight rules issued by the country for aircrafts flying in the sky. The main purpose is to maintain flight order and prevent collision of aircrafts and collision between aircrafts with barriers on the ground. Air traffic control is one way for various countries in the world to manage their own territorial air space and there are clear legislation and provisions in general. Civil aviation companies in our country have specific provisions for use range of territorial air space and the air space exceeding scope of civil aviation will be under management of national defense troops in our country-air force organization of the Chinese People's Liberation Army.

\section{Modeling assigned for decision making of air traffic control}

Basic needs of air traffic control center in audio/video commanding and dispatching system: air traffic control center is the central institution of commanding and dispatching and digital switch and transmission technology can be used to provide accurate and efficient audio and video real-time monitoring, commanding and dispatching function for safe flight. It can be used for real-time accurate control and display various data and provide real-time, accurate and efficient informationized and digitized technology guarantee for execution of flight mission. Project design for audio/video commanding and dispatching system of air traffic control center: As for overall system design, the air traffic control center will realize multimedia and audio/video commanding and dispatching system function and design the comprehensive information exchange means based on comprehensive transmission of audio/video, network, text, fax and so on to solve audio/video commanding and dispatching problems "that can be seen, listened and managed", realize accurate and efficient guarantee command of flight mission and provide real-time, accurate and efficient informationized technology guarantee for safe flight mission. The project is named as: "audio/video 
commanding and dispatching system of air traffic control center”.

\section{Decision-making method by decision-making tree based on rough set of complex network}

The theory of RS (Rough set) is one kind of mathematic theory to analyze data which is proposed by Poland mathematician-Z.Pawlak in 1982[9]. After being classified, the theory has defined concepts of fuzziness and uncertainty and it is one kind of new mathematic tool to solve uncertain and incompatible data and inaccuracy problems. In the past several years, numerous knowledge discovery systems based on rough set were developed home and abroad among which the most representative types include LERS (learning from Examples based on Rough Set) system developed by Kanasas University in America, ROSE(Rough Set data Explorer) system developed by Poznan University of Science and Technology in Poland, data analysis system KDD-R of decision-making matrix based on rough set with variable precision and knowledge discovery developed by Regina University in Canada as well as data mining tool ROUGH ENOUGH developed by TrollData Company in Norway.

Definition 1 Decision Table

In form, tetrad $I=(U, R, V, f)$ is a knowledge representation system among which $U$ is the non-null object. $U \times R \rightarrow V$ is an information function and it endows one information value for every attribute of every object. These information values constitute finite set and it is said that domain $\mathrm{R}$ is the non-null finite set of attributes. Thereinto, $\quad V=\bigcup_{r \in R} V_{r}$ and $V_{r}$ are value domain of attribute $r ; f: \forall r \in R$ and $x \in U, f(x, r) \in V$, are generally recorded as $I=(U, R) \mathrm{I}=(\mathrm{U}, \quad \mathrm{R})$ for abbreviation. In case $R=C \cup D$ among which $C$ is the set of condition attributes, $D$ is the set of decision attributes and $C \bigcap D=\varnothing$, then this knowledge representation system is named as decision form.

Definition 2 Equivalence class

In case $I=(U, R)$ and $P \subseteq R$, then $\cap P$ is also an equivalence relation which can be named as undistinguishable relation of $P$ and which is recorded as ind $(P)$ ind $(R)=\left\{(x, y) \in U^{2} \mid \forall a \in P, f(x, a)=f(y, a)\right\}$. All equivalence classess generated from this undistinguishable relation ind $(P)$ can be expressed in $U /$ ind $(P)$ which is recorded as $U / P$ for abbreviation.

Definition 3 Equivalence relation

In case $I=(U, R), P \subseteq R, \quad X \subseteq U, \underline{P}(X)=\left\{x \in U \mid[x]_{P} \subseteq X\right\}$ and $\bar{P}(X)=\left\{x \in U \mid[x]_{P} \cap X \neq \varnothing\right\}$ are named as $P$ lower approximation and upper approximation of $X$. It is obvious that $\underline{P}(X)$ is the set of elements which can be included in X based on knowledge $P$ and $U$ and $\bar{P}(X)$ is the set of elements which can be included in $X$ based on knowledge $P$ and $U$. $\operatorname{pos}_{P}(X)=\underline{P}(X)$ means that $X$ is the positive domain of $R$. In case $P$ and $Q$ are equivalence relation in $U$, then $\operatorname{pos}_{P}(Q)=\bigcup_{x \in U / P} \underline{P}(X)$

Definition 4 Dependency degree

In case $I=(U, R), P, Q \subseteq R$, then when $k=\gamma_{P}(Q)=\frac{\left|\operatorname{pos}_{P}(Q)\right|}{|U|}, Q$ is the dependency degree $P$ of $k(0 \leq k \leq 1)$. When $\mathrm{k}=1$, Q completely depends on $\mathrm{P}$; when $0<\mathrm{k}<1$, Q partly depends on P; when $\mathrm{k}=0$, Q does not depend on P. Thereinto, $|U|$ means the cardinal number of $U$. 
Definition 5 Core and reduction

The set constituted by all necessary attributes in $\mathrm{R}$ is named the core of $\mathrm{R}$ and it is recorded as core $(R)$. In case $\forall r \in R$ is necessary, then $\mathrm{R}$ is independent or $\mathrm{R}$ is dependent. In case $Q \subseteq R$, in case $\mathrm{Q}$ is independent and $U /$ ind $(Q)=U /$ in $d(R)$, then $\mathrm{Q}$ is the reduction of $\mathrm{R}$ or information system I. The relation of core and reduction is: $\operatorname{core}(R)=\bigcap \operatorname{red}(R)$ and the latter of equation means the set of all reductions in attribute set.

Definition 6 Association degree

The tetrad $I=(U, R, V, f)$ is the knowledge representation system among which $R=C \cup D, C$ is the set of condition attribute and $D$ is the set of decision attributes and $C \bigcap D=\varnothing$. In case $V_{D}=\left\{d_{j} \mid j=1,2, \ldots . ., n\right\}$ is the value sequence of decision attribute, $\quad c_{i}=\left\{c_{i j} \mid i=1,2, \ldots . . n ; j=1,2, \ldots . ., n\right\}$ is the value scope of the i condition attribute, then the correlation coefficient between the decision attribute and the $C_{i j}$ object in condition attribute $c_{i}$ can be defined as: $C R_{i}(j)=\frac{\min _{\forall i} \min _{\forall j}\left|d_{j}-c_{i j}\right|+\rho \max _{\forall i} \max _{\forall j}\left|d_{j}-c_{i j}\right|}{\left|d_{j}-c_{i j}\right|+\rho \max _{\forall i} \max _{\forall j}\left|d_{j}-c_{i j}\right|}$

and differentiation parameter is $\rho \in[0,1]$. The association degree of decision attribute $D$ and condition attribute $C_{i}$ can be defined as: $D R_{i}=\frac{1}{n} \sum_{t=1}^{n} C R_{i}(j), i=1,2, \ldots . ., m$.

\section{Experimental analysis of air traffic control decision}

All enterprises and public institutions of civial aviation have developed emergency decision-making system with different software and hardware platforms and the system can be used to assist the decision-making personnel in commanding the rescue in emergency. However, the emergency information accumulated in these systems are expressed in traditional database, file system and other semi-structured and unstructured data and the computer can not understand and deal with it. Management can be simply carried out on emergency information to make system based on traditional database can not efficiently and reasonably organize rescue organization, rescue resources, rescue rehearsal and other emergency information to rapidly prepare emergency decision scheme. Semantic business process management (SBPM) refers to integration of semantic technique (such as noumenon and semantic net) based on traditional business process management (BPM) to provide one business process representation which can be read by machine. In the end, techniques of semantic retrieval and inference are used to improve automatic realization, automatic execution, automatic monitoring and other capacities of business process. The ideology of SBPM is introduced in the Thesis to research how semantization is carried out for emergency rescue business process of civil aviation based on provisions on emergency rescue manageemnt of civil aviation and relevant manuals so as to add structural restraints and provide demantic data and methods support for rapid generation of emergency rescue scheme.

To verify air traffic control model in the Thesis and feasibility of generated scheme, main decision documents of air traffic control model are recorded in Protégé 4.2 and inference engine HermiT 1.3.5 is started for inference and the results。

the results shows part of process chart in inference of AE_BPMNO and the ellipse in imaginery line represents living examples such as task@1 and so on; the ellipse in full line represents category such as rescuingAction and so on; the living examples connected by solid arrow represent the original relation such as the relation between task@1 and task; characters above the arrow represent the relation between living examples, for example, data attribute has_flow_object_name shows the living example A with flow object name is also named living 
example B.

\section{Conclusion}

In the Thesis, theoretical knowledge of rough set is used to analyze decision-making data of several parties in air traffic control and data preprocessing, classification and evaluation, weight value calculation, attribute reduction and a series of processes are carried out to get relatively objective air traffic control results in the end. Compared with traditional air traffic control methods, the method of rough set has overcome disadvantages of subjectivity and one-sidedness of traditional qualitative and quantitative evaluation methods for research on air traffic control. Therefore, more objective and efficient evaluation can be carried out for air traffic control and thus shows relatively stronger practicability.

\section{Reference}

[1] Piecha J, Gnyla P. The transportation network rough description for an adaptive traffic control algorithms by means of video detection technique[J]. Transport Problems An International Scientific Journal, 2013, 8(3):15-24.

[2] Song C S, Sohn Y W, Kwon H J. User Interface Satisfaction of the Air Traffic Control System[J]. 2014, 22(4):1-7.

[3] Adacher L. An agent based approach to the real time air traffic control[C]// World Congress. 2005:2043-2043.

[4] Sadeghzadeh K, Sipahi R. A Predictor-Compensator Design to Assist Human Decision-Making Process in an Air-Traffic-Control Simulator[C]// ASME 2013 Dynamic Systems and Control Conference. 2013:343-9.

[5] Thong D N, Tavanti $\mathrm{M}$, Ha $\mathrm{L} \mathrm{H}$, et al. 3D stereo displays for air traffic control : a multidisciplinary framework and some results[J]. Applied Optics, 2005, 24(22):3766-3772. 